\title{
Study of Impact of Angular Velocity on Ballistic Dispersion
}

\author{
Teng Peng-hua \\ School of Marine Engineering \\ Northwestern Polytechnical University \\ Xi’an, Shaanxi, China, 15091375937 \\ 232810684@qq.com
}

\author{
Zhang Yu-wen \\ School of Marine Engineering \\ Northwestern Polytechnical University \\ Xi'an, Shaanxi, China, 029-88495832 \\ fhzh@pub.xaonline.com
}

\begin{abstract}
Single fin deviation and thrust deviation will cause ballistic dispersion to floating ballistic on carrier. By establishing kinematic equation of underwater six-degree-of-freedom for carrier, it's analyzed that angular velocity of rotation has obvious effect in inhibiting ballistic dispersion, and angular velocity range better inhibiting ballistic dispersion is obtained. Based on this angular velocity range, the design requirement is made for jenkel rudder to produce rotation ability. Stability of motion is taken into account at same time, and $X$-fin rudder is designed. The trajectory simulation is conducted for the carrier added with $X$-fin rudder and simulation result is analyzed. The result shows that the stability of rotary motion of carrier is improved and floating ballistic dispersion is significantly inhibited after $X$-fin rudder is added.
\end{abstract}

Keywords- Angular velocity, $X$-fin rudder, ballistic dispersion, thrust deviation, single fin rudder deviation

\section{INTRODUCTION}

For the underwater carrier under sail, fin rudder will deform at certain extent, causing deviation of single fin rudder. At the same time, certain thrust deviation might occur in the underwater movement for carrier. For both single fin rudder deviation and thrust deviation, floating ballistic dispersion will cause on carrier. For long-distance navigation, ballistic dispersion will produce great deviation for exit point of aircraft and reserved exit point, so that the carrier fails to operate in the designated area. By establishing equation of six degrees of freedom for underwater movement for carrier, the influence of different angular velocity on ballistic dispersion is simulated, to obtain the angular velocity range that can guarantee stable movement of carrier and significantly inhibit ballistic dispersion. Based on the range, the fin rudder of carrier is designed, and numerical simulation is conducted. The simulation results show that angular velocity can significantly inhibit ballistic dispersion of carrier.

\section{ESTABLISHMENT OF EQUATION SET OF SIX DEGREES OF FREEDOM FOR CARRIER}

Establishment of vehicle space motion equation system mainly applies two coordinate systems of body coordinate system oxyz and ground coordinate system $o_{0} x_{0} y_{0} z_{0}$. Body coordinate system is used for establishing vehicle kinetic equation and ground coordinate system is primarily used for determining geometric parameters. The angle of ground coordinate system and body coordinate system determines three attitude angles of vehicle: pitching angle $\theta$, yaw angle $\psi$ and roll angle $\varphi$ and the three coordinates of body coordinate system original point in the ground coordinate system: $x_{0}, y_{0}$ and $z_{0}$ determine spatial position of vehicle.

Establish the following vehicle space motion kinetic equation system in this coordinate system by the use of momentum and principle of momentum moment.

$$
\begin{aligned}
& \left(m+\lambda_{11}\right) \dot{v}_{x}-m y_{c} \dot{\omega}_{z}+m z_{c} \dot{\omega}_{y}+m\left[v_{z} \omega_{y}-v_{y} \omega_{z}-x_{c}\left(\omega_{y}^{2}+\omega_{z}^{2}\right)+y_{c} \omega_{\mathrm{x}} \omega_{\mathrm{y}}+\mathrm{z}_{\mathrm{c}} \omega_{\mathrm{z}} \omega_{\mathrm{x}}\right]=-(G-B) \sin \theta+T+X \\
& \left(m+\lambda_{22}\right) \dot{v}_{y}+\left(m x_{c}+\lambda_{26}\right) \dot{\omega}_{z}-m z_{c} \dot{\omega}_{x}+m\left[v_{x} \omega_{z}-v_{z} \omega_{x}+x_{c} \omega_{x} \omega_{y}+\mathrm{z}_{\mathrm{c}} \omega_{\mathrm{y}} \omega_{\mathrm{z}}-\mathrm{y}_{\mathrm{c}}\left(\omega_{\mathrm{x}}^{2}+\omega_{z}^{2}\right)\right]= \\
& -(G-B) \cos \theta \cos \varphi+Y \\
& \left(m+\lambda_{33}\right) \dot{v}_{z}-\left(m x_{c}-\lambda_{35}\right) \dot{\omega}_{y}+m y_{c} \dot{\omega}_{x}+m\left[v_{y} \omega_{x}-v_{x} \omega_{y}+x_{c} \omega_{z} \omega_{x}+\mathrm{y}_{\mathrm{c}} \omega_{\mathrm{y}} \omega_{\mathrm{z}}-\mathrm{z}_{\mathrm{c}}\left(\omega_{\mathrm{x}}^{2}+\omega_{y}^{2}\right)\right]= \\
& (G-B) \cos \theta \sin \varphi+Z \\
& \left(J_{x x}+\lambda_{44}\right) \dot{\omega}_{x}+m y_{c} \dot{v}_{z}-m z_{c} \dot{v}_{y}+m y_{c}\left(v_{y} \omega_{x}-v_{x} \omega_{y}\right)+m z_{c}\left(v_{z} \omega_{x}-v_{x} \omega_{z}\right)+\left(J_{z z}-J_{y y}\right) \omega_{y} \omega_{z} \\
& =G \cos \theta\left(y_{c} \sin \varphi+z_{c} \cos \varphi\right)-B \cos \theta\left(y_{b} \sin \varphi+z_{b} \cos \varphi\right)+M_{x}
\end{aligned}
$$




$$
\begin{aligned}
& \left(J_{y y}+\lambda_{55}\right) \dot{\omega}_{y}+m z_{c} \dot{v}_{x}-\left(m x_{c}-\lambda_{35}\right) \dot{v}_{z}+m z_{c}\left(v_{z} \omega_{y}-v_{y} \omega_{z}\right)+m x_{c}\left(v_{x} \omega_{y}-v_{y} \omega_{x}\right)+\left(J_{x x}-J_{z z}\right) \omega_{z} \omega_{x}= \\
& -G\left(x_{c} \cos \theta \sin \varphi+z_{c} \sin \varphi\right)+B\left(x_{b} \cos \theta \sin \varphi+z_{b} \sin \varphi\right)+M_{y} \\
& \left(J_{z z}+\lambda_{66}\right) \dot{\omega}_{z}-m y_{c} \dot{v}_{x}+\left(m x_{c}+\lambda_{26}\right) \dot{v}_{y}+m x_{c}\left(v_{x} \omega_{z}-v_{z} \omega_{x}\right)+m y_{c}\left(v_{y} \omega_{z}-v_{z} \omega_{y}\right)\left(J_{y y}-J_{x x}\right) \omega_{x} \omega_{y}= \\
& G\left(y_{c} \sin \theta-x_{c} \cos \theta \cos \varphi\right)-B\left(y_{b} \sin \theta-x_{b} \cos \theta \cos \varphi\right)+M_{z}
\end{aligned}
$$
[1].

The definition of parameters in the equation is same as in

\section{INFLUENCE OF ANGULAR VELOCITY ON BALLISTIC DISPERSION}

Since angular velocity of carrier is produced by differential rudder, the differential steerage takes the place of angular velocity as independent variable for simulation analysis[2][3].

A. The effect simulation of ballistic dispersion caused by angular velocity to inhibition of thrust deflection

Simulation initial condition: (1) $\mathrm{x}_{0}=0 \mathrm{~m}, \mathrm{y}_{0}=-200 \mathrm{~m}, \mathrm{z}_{0}=0 \mathrm{~m}$;

(2) Thrust deflection $\delta_{T}=1^{\circ}, m_{x}^{\delta_{d}}$ change interval 0.033; (3) Carrier speed, angular velocity, attitude angle are $0^{\circ}$.
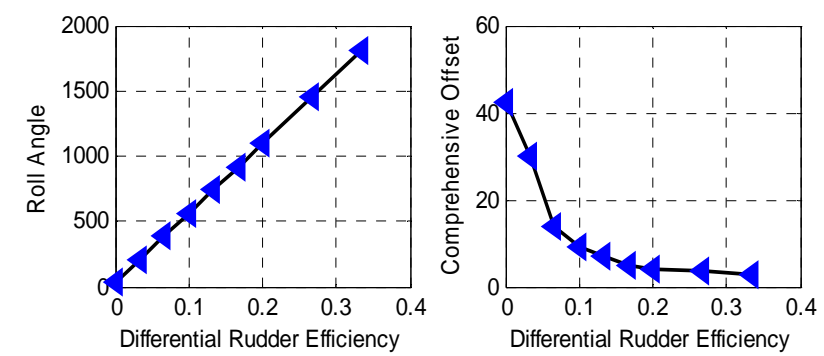

Fig1. Curve of Roll angle and Comprehensive offset along with Differential Rudder Efficiency

The simulation results are shown in Fig 1, giving the change curve of roll angle and comprehensive offset along with differential rudder efficiency.

Analysis of simulation result: (1) The effect of inhibition of thrust deviation increasing with the growing angular velocity of rotation; (2) Effect of inhibition of ballistic dispersion increasing with the reducing angular velocity of rotation, and when $m_{x}^{\delta_{d}}>0.1$, the efficiency inhibiting ballistic dispersion is significantly weakened.

B. The effect simulation of ballistic dispersion caused by angular velocity to inhibition of single fin rudder deviation Simulation initial condition: (1) $\mathrm{x}_{0}=0 \mathrm{~m}, \mathrm{y}_{0}=-200 \mathrm{~m}, \mathrm{z}_{0}=0 \mathrm{~m}$; (2) Left fin deflection $\delta_{h l}=0.1^{\circ}, m_{x}^{\delta_{d}}$ change interval 0.033; (3) Carrier speed, angular velocity, attitude angle are $0^{\circ}$.
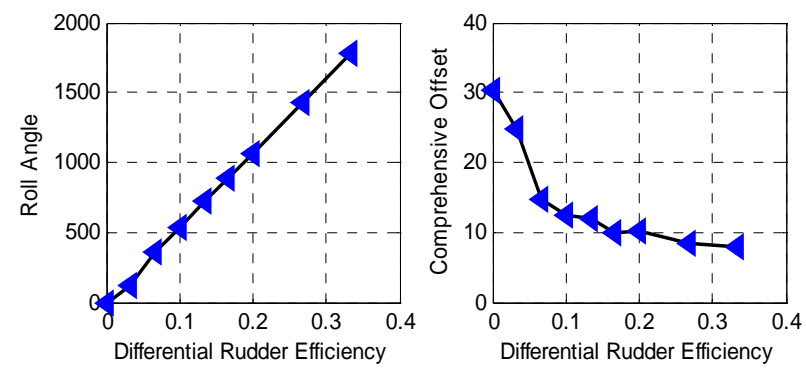

Fig2. Curve of Roll angle and Comprehensive offset along with Differential Rudder Efficiency

The simulation results are shown in Fig 2, giving the change curve of roll angle and comprehensive offset along with differential rudder efficiency.

Analysis of simulation result: (1) The effect of inhibition of thrust deviation increasing with the growing angular velocity of rotation; (2) Effect of inhibition of ballistic dispersion increasing with the reducing angular velocity of rotation; and when $m_{x}^{\delta_{d}}>0.1$, the efficiency inhibiting ballistic dispersion is significantly weakened.

\section{FIN RUDDER DESIGN AND SIMULATION ANALYSIS}

\section{A. Fin rudder design}

The Design of basic idea: (1) The angular velocity of carrier can only use hydrodynamic force, and the most direct and most commonly used way to produce angular velocity is use of differential rudder; (2) From the above simulation results, we can see that the higher angular velocity, the more obvious inhibitory effect. However, in the actual project, to ensure stability of movement, it's impossible to produce too high angular velocity. Rotation angle $560^{\circ} \sim 720^{\circ}$ is the best; (3) The set differential rudder angle should not be too big; otherwise, the navigation resistance will significantly increase. The angle is generally not more than $5^{\circ}[4][5]$.

Based on the above ideas, the design of fin rudder shall satisfy the following requirements: (1) A simple operation can achieve the control of fin rudder, occupy rudder angle as little as possible, and set aside sufficient rudder angle to control carrier; (2) Because carrier shall make rotating movement, the distribution of fin rudder shall enhance the stability of rotary motion; (3) Differential rudder angle shall be kept within $4^{\circ} \sim 5^{\circ}$, not too large[6].

The rudder of original carrier shall not be used for differential angle. In addition, four rudder tablets are designed to allow it to distribute in $X$ shape, as in Fig 3. 


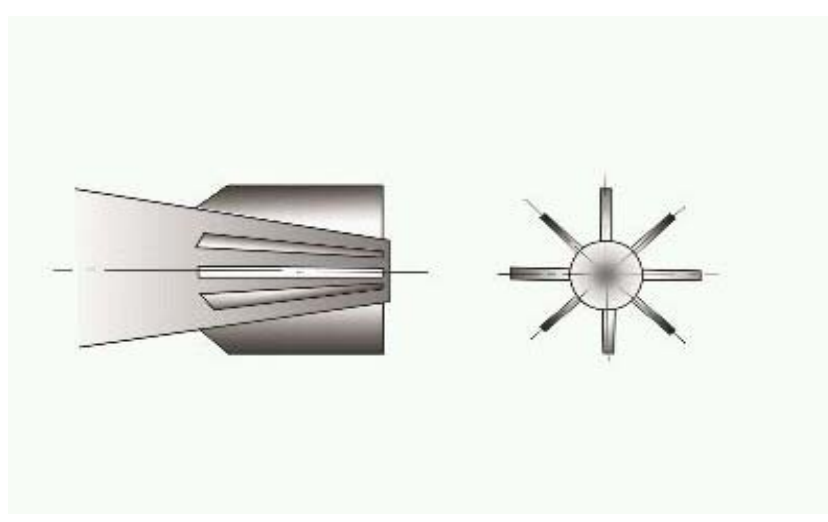

Fig3. $X$-shape Jenkel Rudder

\section{B. Simulation analysis}

1) Simulation of influence inhibiting thrust declination

Simulation initial condition: (1) $\mathrm{x}_{0}=0 \mathrm{~m}, \mathrm{y}_{0}=-200 \mathrm{~m}, \mathrm{z}_{0}=0 \mathrm{~m}$; (2) Thrust angle $\delta_{T}=1^{\circ}$, differential rudder change interval is $1^{\circ}$; (3) Carrier speed, angular velocity, attitude angle are $0^{\circ}$.

Simulation results are shown in Table1.

2) Simulation of influence inhibiting single fin rudder angle

Simulation initial condition: (1) $\mathrm{x}_{0}=0 \mathrm{~m}, \mathrm{y}_{0}=-200 \mathrm{~m}, \mathrm{z}_{0}=0 \mathrm{~m}$; (2) Left fin angle $\delta_{h l}=0.4^{\circ}$, differential rudder change interval is $1^{\circ}$; (3) Carrier speed, angular velocity, attitude angle are $0^{\circ}$.

Simulation results are shown in Table 2.

\section{Analysis of simulation results}

Result of analysis of simulation data in Table 1 and Table 2: (1) Carrier's rotary motion can significantly inhibit floating ballistic disperse caused by thrust deviation and single fin rudder deviation; (2) Data in Table 1 show that when differential rudder angle $\delta_{d}$ is $2^{\circ}$, comprehensive offset reduces significantly. With increased differential rudder angle, comprehensive offset is not significantly changed. It indicates that the increase of $X$-shape differential rudder significantly inhibits floating ballistic dispersion of carrier and increases stability of rotary motion; (3) The data in Table 2 show that with the increasing differential rudder angle $\delta_{d}$, comprehensive offset reduces continuously,. When differential rudder angle is $4^{\circ}$, offset decreases by $95 \%$.

TABLE1. RESULTS OF INFLUENCE INHIBITING THRUST DECLINATION

\begin{tabular}{|l|l|l|l|l|l|l|}
\hline$m_{x}^{\delta_{d}}$ & $\delta_{d}(\mathrm{deg})$ & $\varphi(\mathrm{deg})$ & $\begin{array}{c}\text { effluent } \\
\text { time }\end{array}$ & $y(m)$ & $z(m)$ & $\begin{array}{l}\text { offset } \\
\text { volume }\end{array}$ \\
\hline 0 & 0 & 0 & 7.686 & 0 & -29.2 & 29.2 \\
\hline 0.1 & 0 & 0 & 8.193 & 0 & -11.4 & 11.4 \\
\hline 0.1 & 1 & 195.9 & 8.192 & 5.24 & -7.28 & 8.98 \\
\hline 0.1 & 2 & 391.6 & 8.184 & 3.34 & -3.14 & 8.184 \\
\hline
\end{tabular}

\begin{tabular}{|l|l|l|l|l|l|l|}
\hline 0.1 & 3 & 587.3 & 8.171 & 2.17 & -3.92 & 8.171 \\
\hline 0.1 & 4 & 783 & 8.17 & 2.49 & -3.32 & 8.17 \\
\hline
\end{tabular}

TABLE2. RESULTS OF INFLUENCE INHIBITING SINGLE FIN RUDDER ANGLE

\begin{tabular}{|l|l|l|l|l|l|l|}
\hline$m_{x}^{\delta_{d}}$ & $\delta_{d}(\mathrm{deg}$ & $\varphi(\mathrm{deg})$ & $\begin{array}{c}\text { effluent } \\
\text { time }\end{array}$ & $y(m)$ & $z(m)$ & $\begin{array}{l}\text { offset } \\
\text { volume }\end{array}$ \\
\hline 0 & 0 & 30.41 & 8.029 & -41.2 & -6.67 & 42.25 \\
\hline 0.1 & 0 & 127.1 & 8.247 & -15.5 & -2.43 & 15.72 \\
\hline 0.1 & 1 & 223.1 & 8.203 & -3.66 & -9.74 & 10.42 \\
\hline 0.1 & 2 & 418.5 & 8.181 & -0.07 & -3.98 & 3.988 \\
\hline 0.1 & 3 & 614.1 & 8.173 & -0.06 & -3.31 & 3.319 \\
\hline 0.1 & 4 & 809.7 & 8.163 & 0.19 & -1.93 & 1.944 \\
\hline
\end{tabular}

\section{CONCLUSION}

By establishing kinematic equation of underwater six-degree-of-freedom for carrier, the influence of angular velocity on floating ballistic dispersion is analyzed, and carrier can significantly inhibit ballistic dispersion through rotary motion. Combined with actual situation of carrier navigation, eight fin rudders in $X$-shape are designed, accompanied by ballistic simulation analysis. The analysis result shows that after the carrier increases fin rudder of $X$-shape distribution, the stability of rotary motion is enhanced and rotational motion also obviously inhibits floating ballistic dispersion.

\section{REFERENCES}

[1] Zhang $\mathrm{Yu}$ wen, Torpeto trajectory and trajectory design[M], Northwestern Polytechnical University Publishing Company, China, p. 42 72, 1996

[2] Confe G, Serrani A, "odeling and simulation of underwater vehicles," Poceedings of the 1996 IEEE International Symposium on Computer Aided Control System Design, 1996: 1518.

[3] Cenko A, Kraing K, Tseng W, “Application to Theoretical Trajectory Predictions," AIAA 87-0210.

[4] S.M Smith, "Stability and Dispersion Analysis for Rockets and Projectiles”, 1970, 9 21.

[5] Prestero, "Development of a six degree of freedom simulation model for the REMUS autonmous underwater vehicle,” MTS/IEEE Oceans 2001. An Ocean odyssey. Conference Proceedings, 2001, vol1, 450 455

[6] Humphreys,D.E, "Dynamics and Hydrodynamics of ocean vehicles,” In Proceedings MTS/IEEE, Oceans 2000.

[7] http://www.ise.bc.ca/, Internation Submarine Engineering Ltd. 